\title{
Tramadol/paracetamol combination tablet for postoperative pain following ambulatory hand surgery: a double-blind, double-dummy, randomized, parallel-group trial
}

\author{
This article was published in the following Dove Press journal: \\ Journal of Pain Research \\ 7 April 2011 \\ Number of times this article has been viewed
}

\author{
Narinder Rawal' \\ Valery Macquaire ${ }^{2}$ \\ Elena Catalá ${ }^{3}$ \\ Marco Berti ${ }^{4}$ \\ Rui Costa ${ }^{5}$ \\ Markus Wietlisbach ${ }^{6}$ \\ 'Department of Anesthesiology \\ and Intensive Care, Örebro \\ University Hospital, Örebro, Sweden; \\ ${ }^{2}$ Clinique du Parc Leopold, Brussels, \\ Belgium; ${ }^{3}$ Pain Clinic, Department \\ Anesthesiology, Hospital de la Santa \\ Creu i Sant Pau, Barcelona, Spain; \\ ${ }^{4}$ Department of Anesthesiology and \\ Reanimation, Parma Hospital, Parma, \\ Italy; ${ }^{5}$ Garcia de Orta Hospital, \\ Almada, Portugal; ${ }^{6}$ Department of \\ Anesthesiology, Sursee Hospital, \\ Sursee, Switzerland
}

\begin{abstract}
This randomized, double-blind, double-dummy, multicenter trial compared efficacy and safety of tramadol HCL $37.5 \mathrm{mg} /$ paracetamol $325 \mathrm{mg}$ combination tablet with tramadol HCL $50 \mathrm{mg}$ capsule in the treatment of postoperative pain following ambulatory hand surgery with iv regional anesthesia. Patients received trial medication at admission, immediately after surgery, and every 6 hours after discharge until midnight of the first postoperative day. Analgesic efficacy was assessed by patients $(n=128$ in each group, full analysis set) and recorded in a diary on the evening of surgery day and of the first postoperative day. They also documented the occurrence of adverse events. By the end of the first postoperative day, the proportion of treatment responders based on treatment satisfaction (primary efficacy variable) was comparable between the groups ( $78.1 \%$ combination, $71.9 \%$ tramadol; $P=0.24$ ) and mean pain intensity (rated on a numerical scale from $0=$ no pain to $10=$ worst imaginable pain) had been reduced to $1.7 \pm 2.0$ for both groups. Under both treatments, twice as many patients experienced no pain $($ score $=0)$ on the first postoperative day compared to the day of surgery $(35.9 \%$ vs $16.4 \%$ for tramadol/paracetamol and $36.7 \%$ vs $18 \%$ for tramadol treatment). Rescue medication leading to withdrawal (diclofenac $50 \mathrm{mg}$ ) was required by $17.2 \%$ patients with tramadol/paracetamol and $13.3 \%$ with tramadol. Adverse events (mainly nausea, dizziness, somnolence, vomiting, and increased sweating) occurred less frequently in patients under combination treatment $(P=0.004)$. Tramadol/paracetamol combination tablets provided comparable analgesic efficacy with a better safety profile to tramadol capsules in patients experiencing postoperative pain following ambulatory hand surgery.
\end{abstract}

Keywords: ambulatory hand surgery, analgesia, combination therapy, paracetamol, postoperative pain, tramadol

\section{Introduction}

The development of minimally invasive surgical procedures and an improvement in anesthetic techniques have resulted in a continuous world-wide increase in ambulatory (outpatient) surgery. ${ }^{1,2}$ Although most ambulatory procedures are associated with relatively minor surgical trauma, severe postoperative pain can occur causing extreme discomfort, sleep deprivation and suffering ${ }^{3}$ which might delay discharge or cause unanticipated hospital admission, thereby increasing the burden for the patients as well as healthcare costs. ${ }^{4,5}$ The proportion of patients experiencing moderate to severe pain in the first 24 to 48 postoperative hours can reach $40 \% .{ }^{6,7}$ Appropriate postoperative pain management is therefore indicated.
Correspondence: Narinder Rawal Department of Anesthesiology and Intensive Care, Örebro University Hospital, 70185 Örebro, Sweden Tel +46 196021000 Fax +46 I9602।I26

Email narendra.rawal@orebroll.se 
In recent years, pain management has focused on the therapeutic potential of combining analgesic medications with complementary pharmacokinetic and pharmacodynamic profiles to achieve greater efficacy and a better safety profile than the individual agents. ${ }^{8,9}$ In the fixed-combination tablet tramadol HCL $37.5 \mathrm{mg} /$ paracetamol $325 \mathrm{mg}$ (Grünenthal GmbH, Aachen, Germany), the two individual components have different mechanisms of action. Tramadol HCL (Grünenthal $\mathrm{GmbH}$ ), a centrally acting weak opioid agonist (with selectivity for the $\mu$-receptor) and monoamine neurotransmitter reuptake inhibitor, has been proven to provide sustained relief for moderate to severe postoperative pain for several types of surgery ${ }^{10}$ whereas paracetamol, a rapid-onset, nonopioid analgesic and antipyretic, ${ }^{11}$ is used for the management of mild to moderate pain. The combination tablet was proven efficacious in acute postoperative dental pain, ${ }^{12}$ postoperative pain following orthopedic and abdominal surgery, ${ }^{13}$ fibromyalgia pain, ${ }^{14}$ low back pain, ${ }^{15-17}$ migraine pain, ${ }^{18}$ and as add-on therapy for osteoarthritis flare ${ }^{19}$ and rheumatoid arthritis pain. ${ }^{20} \mathrm{~A}$ detailed review of the use of fixed-dose tramadol/paracetamol in the management of moderate to severe pain has been published. ${ }^{21}$ In addition, it was suggested in a recent study that the combination tablet might be as effective as gabapentin in the treatment of painful diabetic neuropathy in patients with type 2 diabetes. ${ }^{22}$

Hand surgery is frequently performed on an outpatient basis. In many cases, regional anesthetic techniques including brachial plexus or major nerve blocks with intravenous anesthesia are used, ${ }^{23,24}$ thereby avoiding complications observed with general anesthesia. However, postoperative pain management at home using either tramadol, metamizol, or paracetamol as single substances after ambulatory hand surgery has been shown to be inadequate for up to $40 \%$ of all patients in a controlled trial. ${ }^{25}$ The objective of the present trial was to compare efficacy and tolerability between the combination analgesic tramadol $\mathrm{HCl} 37.5 \mathrm{mg} /$ paracetamol $325 \mathrm{mg}$ (tramadol/paracetamol) and tramadol HCL $50 \mathrm{mg}$ (tramadol) monotherapy in the management of postoperative pain following ambulatory hand surgery.

\section{Methods}

\section{Patients}

Patients between 18 and 75 years of age in good physical health according to the American Society of Anesthesiologists scale (ASA stage I or II) ${ }^{26}$ were included if they presented with a pathological condition involving bony or ligamentous structures of the hand requiring surgical intervention. Main exclusion criteria were intake of short-acting analgesics within 12 hours prior to surgery until the final visit (second postoperative day) except for oral diclofenac $50 \mathrm{mg}$ for rescue and after 24:00 h of the second calendar day; intake of long-acting analgesics within 72 hours prior to surgery until the final visit; intake of monoamine oxidase inhibitors within 30 days prior to surgery until 72 hours after the final visit; serious psychiatric or neurologic disorders, in particular epilepsy; known hypersensitivity to tramadol, paracetamol, or nonsteroidal anti-inflammatory medication; and a risk in terms of precautions, warnings, and contraindications in the product monograph for tramadol, tramadol/paracetamol, or diclofenac. Pregnant or lactating females were not allowed to participate.

Medication for concomitant diseases could be taken provided the patient had been on a stable dose for 30 days prior to surgery. For the symptomatic treatment of nausea and vomiting metoclopramide $10 \mathrm{mg}$ up to a maximum daily dose of $30 \mathrm{mg}$ was recommended.

\section{Trial design}

This double-blind, double-dummy, randomized, parallelgroup trial was conducted from April to October 2003 according to the Declaration of Helsinki and Good Clinical Practice at 24 trial centers in 9 European countries (Belgium, France, Germany, Italy, Portugal, Spain, Sweden, Switzerland, and the Netherlands). Trial protocol and amendments were approved by the corresponding independent Ethics Committees of each participating country; patients' written informed consent was obtained at enrolment.

Prior to hand surgery, patients were randomly assigned in a 1:1 ratio to receive either tramadol/paracetamol tablets plus placebo capsules or tramadol capsules plus placebo tablets (double-dummy design). Placebo capsules and tablets were identical in size and appearance to the active medication. Computer-generated lists for blockwise randomization following the treatment allocation ratio of 1:1 were prepared by an independent clinical research organization (M.A.R.C.O., Düsseldorf, Germany) and block size was given in the randomization list but not to the investigators; investigators and patients were blinded to the treatment.

Surgical procedures were carried out as ambulatory surgery. Patients received 1 to $3 \mathrm{mg}$ midazolam intravenously as premedication. Intravenous regional anesthesia was performed as follows: a canula was inserted in the dorsum of the hand on the side to be operated on. An inflatable doublecuff tourniquet with padding was applied to the upper arm. A tight elastic bandage was wrapped from distal to proximal while the arm was elevated. Alternatively, if the bandaging 
was too painful for the subject to tolerate, the arm could be elevated for 3 minutes. The distal cuff was first inflated followed by the proximal cuff. The distal cuff was then deflated. The cuffs were inflated to $100 \mathrm{mmHg}$ above the subject's systolic pressure (pulse occlusion could be determined by oximeter). Next, the bandage was removed and the absence of a pulse was confirmed. A local anesthetic (lidocaine $3 \mathrm{mg} /$ $\mathrm{kg}$ or prilocaine $3 \mathrm{mg} / \mathrm{kg}$ ) was injected using the dorsal hand canula. The injection was performed slowly at a rate of approximately $0.5 \mathrm{~mL} / \mathrm{sec}$. If the compression by the proximal cuff caused pain during surgery, the distal cuff was inflated followed by deflation of the proximal cuff.

Independently of the duration of surgery, the double-cuff tourniquet was to remain inflated for at least 20 minutes after the injection. The maximum time of inflation should not exceed 90 minutes. The subject was observed and vital signs were recorded immediately before and for 10 minutes after deflation of the tourniquet. Sedation was assessed before the second measurement of the vital signs. Intraoperative sedation with midazolam was allowed but the total dose, including pre-medication, should not exceed $7.5 \mathrm{mg}$.

The trial included a surgery day and a postoperative day. Upon discharge on surgery day, patients were given a diary to document their efficacy and safety evaluations at home. On the second postoperative day, patients visited the center for final assessments and returned the diary. Trial medication ( 1 active unit plus 1 placebo unit) was first administered at admission; a second dose was given in the recovery room after assessment of vital signs and sedation 10 minutes after deflation of the tourniquet. At home, patients took 1 unit of active plus 1 unit of placebo trial medication every 6 hours (except at nighttime if not required); an additional unit could be taken in case of insufficient analgesia (earliest 30 minutes after the regular dose). The maximum permitted daily dose was 8 tablets/capsules of active medication. Patients were instructed not to take any trial medication after midnight of the first postoperative day. No other analgesic medication was permitted. If the patient wished to discontinue the trial medication owing to an adverse event or lack of efficacy, diclofenac $50 \mathrm{mg}$ (not exceeding $150 \mathrm{mg}$ daily) could be used as rescue medication.

\section{Outcome variables}

Pain intensity was assessed by the patient after surgery (10 minutes after deflation of the tourniquet). Further efficacy assessments were performed by the patient at home and recorded in a diary on the evenings of the surgery and the first postoperative day. All entries were to be completed before midnight of the first postoperative day; information on treatment response and pain intensity was to be recorded immediately before bedtime.

The primary efficacy variable was the rate of patients responding to treatment based on treatment satisfaction as recorded on the evening of the first postoperative day using a 4 -point verbal rating scale $(0=$ poor, $1=$ fair, $2=$ good, 3 = excellent). A patient was defined as a responder with a rating of 2 or 3 and no intake of rescue medication or other concomitant analgesia before midnight of the first postoperative day.

Secondary efficacy variables included the assessment of the patient's average pain intensity over the last 24 hours for surgery and first postoperative day on an 11-point numerical rating scale (from $0=$ no pain to $10=$ worst imaginable pain), time to first intake and total intake of trial medication at home, time to discontinuation and premature discontinuation rate owing to lack of efficacy, time to first intake and total intake of rescue medication, and total intake of antiemetic medication. For premature discontinuation due to lack of efficacy, intake of rescue medication or any other analgesic medication was taken as time point of termination.

Safety evaluations by the investigator included adverse events (AEs) documentation and sedation assessment after surgery (10 minutes after deflation of the tourniquet) and before discharge, and vital signs (pulse and respiratory rate, and systolic and diastolic blood pressure) at admission, immediately before and 10 minutes after tourniquet deflation, and before discharge. AEs were also recorded by the patients. Additionally, premature discontinuation owing to AEs and the time to this event were documented.

\section{Statistical analysis}

In order to detect a difference in response rates (based on the patients' treatment satisfaction) of at least $20 \%$ between the two treatment groups and assuming a 50\% response rate for tramadol, it was determined that a sample size of 116 patients per group was required to achieve an appropriate statistical power of $85 \%$. Taking a $10 \%$ drop-out rate into account, a total of 258 patients was required in order to obtain 232 patients completing the trial.

Efficacy was analyzed using the full analysis set (FAS) which included all randomized patients who received at least 1 dose of trial medication and provided information with respect to the primary efficacy variable. A per-protocol (PP) analysis including all FAS patients without major protocol violations was used for sensitivity analysis. 
All statistical tests were performed as 2-sided tests with a significance level of 5 unless otherwise specified. All statistical analysis was done with pooled centers, the exception being the primary variable which was also evaluated per center. Response rates were analyzed using a Cochran-Mantel-Haenszel (CMH) test adjusting for center; the interaction of treatment and center was checked by a Breslow-Day test for homogeneity. The difference between treatment groups was calculated using the associated 2-sided 95\% confidence interval (CI). Treatment differences were considered significant at $P<0.05$. The primary analysis was the only confirmatory analysis; all secondary analyses were exploratory. Treatment differences for the parameter pain intensity as assessed after surgery were analyzed by means of an analysis of variance including effects for treatment, center, and treatment and center interaction. A separate analysis of variance was carried out for the assessment by the patients at home. A stratified Cox proportional hazards model using treatment as factor and center as stratum was applied for the analyses of time to premature trial discontinuation due to AEs and due to lack of efficacy. The last observation carried forward approach was used only for the assessment of the primary variable, the secondary efficacy variable pain intensity, and the safety variable sedation. If the patient's assessment of the primary variable for the first postoperative day was missing, the assessment on the evening of surgery day was used. If the assessment from the operative day was also missing, this value was replaced by 'poor'.

All patients receiving at least 1 dose of trial medication were included in the descriptive safety analysis. Adverse events were encoded using the Medical Dictionary for Regulatory Activities (MedDRA) version 6.0.

All statistical analyses were performed with SAS version 8.2 (SAS Institute, Cary, NC, USA).

\section{Results}

A total of 132 patients received tramadol/paracetamol combination treatment, and 129 patients took tramadol. The majority completed the trial $(83.5 \%)$; reasons for premature withdrawal were lack of efficacy, AEs, or protocol violations (Figure 1).

Most patients $(98 \%)$ were included in the primary FAS analysis; 39 of those (21 in the tramadol, 18 in the tramadol/ paracetamol group) were excluded from the PP analysis $(n=217)$ due to violations of the study protocol. Baseline characteristics and surgery details were comparable between both groups (FAS population; Table 1). More females than

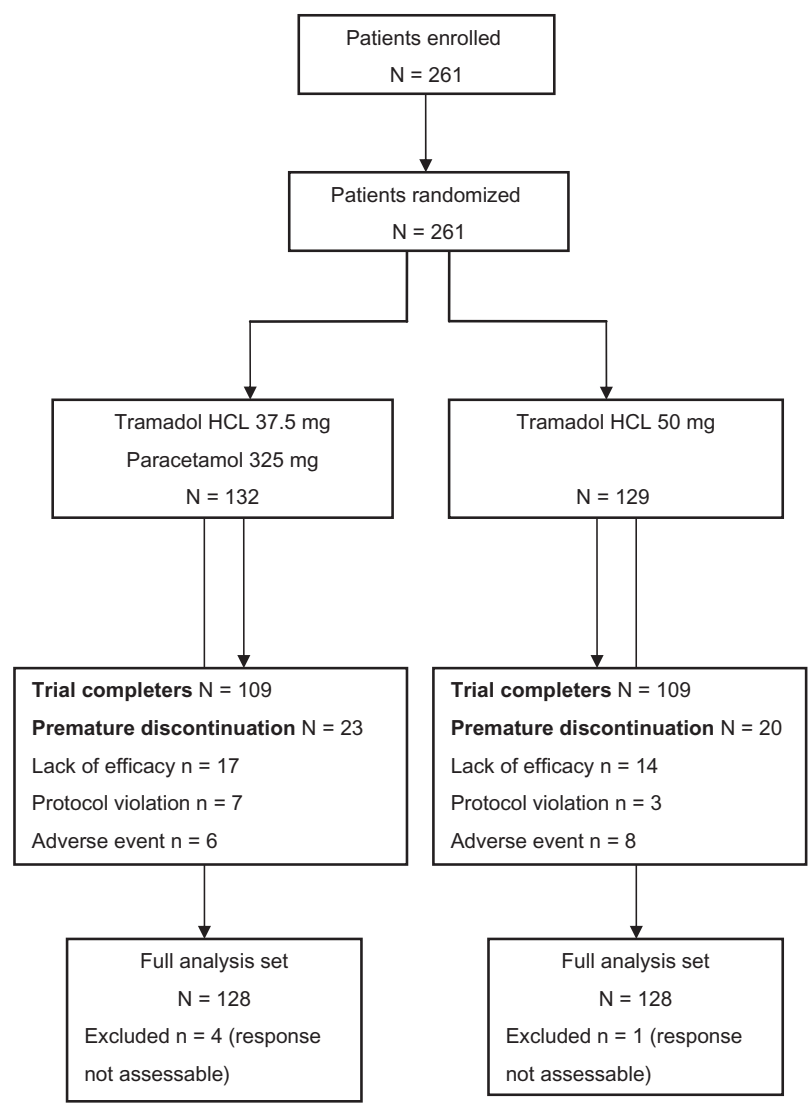

Figure I CONSORT flow chart.

males participated; except for 4 tramadol-treated patients, the trial population was of Caucasian origin.

\section{Efficacy outcomes}

\section{Treatment satisfaction}

A larger proportion of FAS patients in the tramadol/ paracetamol group (78.1\% vs $71.9 \%$ in the tramadol group) were considered treatment responders at the end of the first postoperative day (primary efficacy variable). The estimated difference in response rate of $6.25 \%$ (95\% CI $[-4.3,16.8]$; $P=0.24[\mathrm{CMH}$ test $]$ ) in favor of tramadol/paracetamol treatment was, however, statistically not significant. The PP analysis confirmed the finding ( $83.6 \%$ vs $75.7 \%$ ), with an estimated difference of $7.94 \%$ (tramadol/paracetamol vs tramadol; 95\% CI $[-2.7,18.6] ; P=0.13$ [CMH test]).

Treatment satisfaction was also recorded as at least good in more patients on combination therapy ( $77.3 \%$ vs $71.9 \%$ on tramadol treatment) for the day of surgery (Figure 2); again differences were not significant.

\section{Pain intensity}

Pain intensity was comparable for both treatments. Ten minutes after deflation of the tourniquet, mean pain 
Table I Baseline characteristics and surgery details of the trial population (full analysis set)

\begin{tabular}{|c|c|c|}
\hline & $\begin{array}{l}\text { Tramadol/ } \\
\text { paracetamol } \\
(n=I 28)\end{array}$ & $\begin{array}{l}\text { Tramadol } \\
(n=128)\end{array}$ \\
\hline Gender, male/female (\%) & $41.4 / 58.6$ & $35.9 / 64.1$ \\
\hline Age (years) & $46.2 \pm 13.4$ & $47.6 \pm 12.9$ \\
\hline Body mass index $\left(\mathrm{kg} / \mathrm{m}^{2}\right)$ & $26.3 \pm 4.8$ & $26.4 \pm 5.3$ \\
\hline \multicolumn{3}{|l|}{ Surgery details } \\
\hline \multicolumn{3}{|l|}{ Type of hand surgery } \\
\hline Bony & $21(16.4 \%)$ & $21(16.4 \%)$ \\
\hline Ligamentous & $107(83.6 \%)$ & $107(83.6 \%)$ \\
\hline \multicolumn{3}{|l|}{ Dose of local anesthesia during surgery } \\
\hline \multicolumn{3}{|l|}{ Lidocaine } \\
\hline Total & $198.4 \pm 55.6$ & $199.6 \pm 81.2$ \\
\hline Per kg body weight & $2.7 \pm 0.6$ & $2.7 \pm 1.2$ \\
\hline \multicolumn{3}{|l|}{ Prilocaine } \\
\hline Total & $285.0 \pm 89.3$ & $272.1 \pm 93.2$ \\
\hline Per kg body weight & $4.0 \pm 1.5$ & $3.7 \pm 1.3$ \\
\hline Duration of surgery $(\mathrm{min})$ & $26.1 \pm 14.4$ & $24.8 \pm 14.4$ \\
\hline Duration of tourniquet inflation (min) & $44.5 \pm 15.6$ & $43.8 \pm 16.7$ \\
\hline Total dose of midazolam (mg) & $3.1 \pm 1.8$ & $3.2 \pm 2.0$ \\
\hline $\begin{array}{l}\text { Pain intensity } 10 \mathrm{~min} \text { after tourniquet } \\
\text { deflation }\end{array}$ & $2.7 \pm 2.2$ & $2.9 \pm 2.6$ \\
\hline
\end{tabular}

Note: Data are mean \pm SD or number of patients (\%).

intensity was $2.7 \pm 2.2$ for patients on tramadol/paracetamol and $2.9 \pm 2.6$ for patients treated with tramadol, and remained on a similar mean score on the evening of surgery day $(2.6 \pm 2.2$ vs $2.8 \pm 2.2$; ANOVA $P=0.47)$. A total of $71.9 \%$ of tramadol/paracetamol and $64.1 \%$ of tramadol patients rated pain intensity with a score of $\leq 3$ on the 11-point NRS on the evening of surgery day. The proportion of patients with this score increased to $83.6 \%$ of tramadol/paracetamol and $81.3 \%$ of tramadol patients in the evening of the first postoperative day which corresponded to mean scores of $1.7 \pm 2.0$ for both groups (ANOVA $P=0.61$ ). The proportion of patients experiencing no pain (score $=0$ ) on the evening of the first postoperative day had markedly increased from surgery day: $16.4 \%$ to $35.9 \%$ for tramadol/paracetamol, $18 \%$ to $36.7 \%$ for tramadol treatment.

\section{Further assessments}

Table 2 lists further assessment outcomes. The intake of trial medication was comparable for the treatment groups; $80.5 \%$ in the tramadol/paracetamol and $82 \%$ in the tramadol group reported a total intake of 4 to 8 tablets or capsules. In total, the group on combination treatment received $29.55 \mathrm{~g}$ of tramadol, whereas the group on tramadol monotherapy received $38.8 \mathrm{~g}$. This amounts to a reduction in tramadol intake of $23.8 \%$ using combination treatment. Time to first intake of trial medication was also comparable between the treatment groups.
Day of surgery

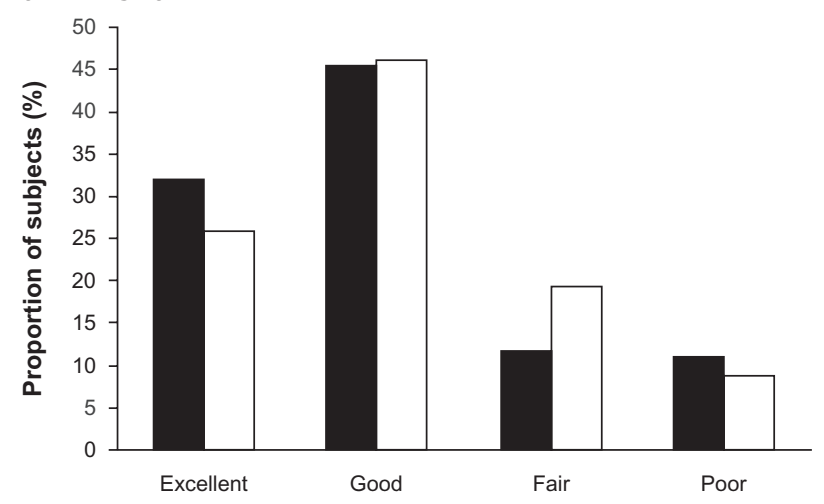

First postoperative day

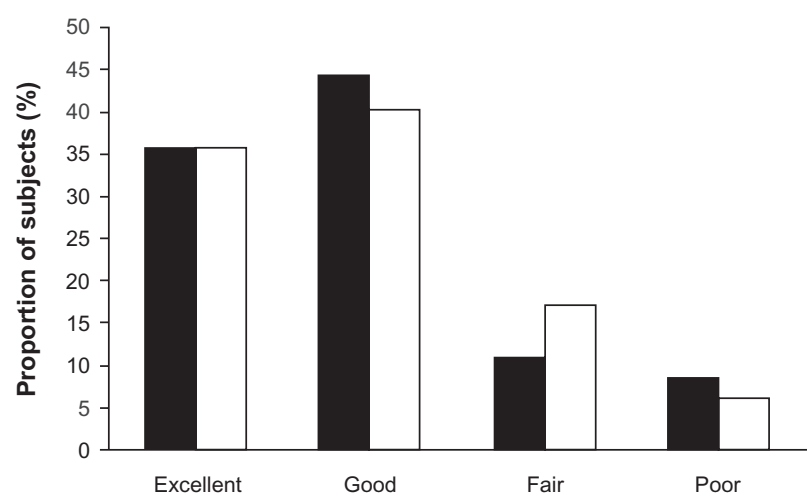

Figure 2 Treatment satisfaction as recorded by the trial population on the evening of surgery and of the first postoperative day (full analysis set). (অ) tramadol/ paracetamol combination therapy, $(\square)$ tramadol monotherapy.

Table 2 Additional secondary efficacy outcomes (full analysis set)

\begin{tabular}{|c|c|c|}
\hline & $\begin{array}{l}\text { Tramadol/ } \\
\text { paracetamol } \\
(\mathbf{n}=\mathbf{I} 28)\end{array}$ & $\begin{array}{l}\text { Tramadol } \\
(n=\mid 28)\end{array}$ \\
\hline \multicolumn{3}{|l|}{ Number of tablets/capsules taken } \\
\hline$\leq 2$ & $4(3.1 \%)$ & $5(3.9 \%)$ \\
\hline 3 & $12(9.4 \%)$ & $9(7 \%)$ \\
\hline 4 & $11(8.6 \%)$ & $17(13.3 \%)$ \\
\hline 5 & $12(9.4 \%)$ & $14(10.9 \%)$ \\
\hline 6 & $29(22.7 \%)$ & $29(22.7 \%)$ \\
\hline 7 & $4 \mathrm{I}(32 \%)$ & $3 \mathrm{I}(24.2 \%)$ \\
\hline 8 & $10(7.8 \%)$ & $14(10.9 \%)$ \\
\hline$>8$ & $9(7 \%)$ & $9(7 \%)$ \\
\hline $\begin{array}{l}\text { Time to first intake of trial } \\
\text { medication }(\mathrm{h})\end{array}$ & $6.59 \pm 1.82$ & $6.73 \pm 2.04$ \\
\hline Rescue medication (diclofenac $50 \mathrm{mg}$ ) & $22(17.2 \%)$ & $17(13.3 \%)$ \\
\hline $\begin{array}{l}\text { Time to first intake of rescue } \\
\text { medication }(h)\end{array}$ & $25.6 \pm 20.8$ & $25.6 \pm 20.0$ \\
\hline $\begin{array}{l}\text { Antiemetic medication } \\
\text { (metoclopramide) }\end{array}$ & $21(16.4 \%)$ & $28(21.9 \%)$ \\
\hline $\begin{array}{l}\text { Premature withdrawal due to lack } \\
\text { of efficacy }\end{array}$ & $17(13.3 \%)$ & $14(10.9 \%)$ \\
\hline $\begin{array}{l}\text { Time to premature withdrawal due } \\
\text { to lack of efficacy (h) }\end{array}$ & $12.8 \pm|1| .3$ & $10.2 \pm 9.0$ \\
\hline
\end{tabular}

Note: Data are mean \pm SD or number of patients (\%). 
More patients on combination therapy (17.2\% vs $13.3 \%$ for monotherapy) used rescue medication; the difference was, however, not significant. There were also no significant differences between treatments for any of the other secondary efficacy variables.

\section{Safety outcomes}

The incidence of treatment-emergent AEs differed significantly between the groups: 54 (40.9\%) patients on tramadol/ paracetamol reported 130 AEs whereas 74 (57.4\%) tramadol patients experienced 193 AEs (explorative $P=0.0041$; difference: $-16.5 \%$ [95\% CI: $-28.4,-4.5]$ ). Most AEs were mild to moderate in intensity. Fourteen and $17 \mathrm{AEs}$ of severe intensity were observed in patients receiving combination therapy and patients on tramadol, respectively, mostly nausea, vomiting and dizziness. Table 3 lists all AEs with a frequency of at least $5 \%$ in either group.

Nausea was the most common event in both groups $(25.8 \%$ for tramadol/paracetamol vs $36.4 \%$ for tramadol) followed by dizziness (15.9\% vs $18.6 \%$ ) and somnolence (9.1\% vs $14 \%$ ). Overall, incidences were lower in the tramadol/paracetamol group for gastrointestinal disorders $(28.8 \%$ vs $44.2 \%$ for tramadol) and nervous system disorders (21.2\% vs $32.6 \%)$. For combination treatment, $110(84.6 \%)$ of the AEs were considered at least possibly related to the trial medication; the corresponding numbers for tramadol monotherapy were 170 (88.1\%).

Table 3 Summary of treatment-emergent adverse events (safety population)

\begin{tabular}{|c|c|c|}
\hline & $\begin{array}{l}\text { Tramadol/ } \\
\text { paracetamol } \\
(n=132)\end{array}$ & $\begin{array}{l}\text { Tramadol } \\
(n=129)\end{array}$ \\
\hline Any adverse event & $54(40.9 \%)$ & $74(57.4 \%)$ \\
\hline Any severe adverse event & $8(6.1 \%)$ & $7(5.4 \%)$ \\
\hline Any serious adverse event & I (0.8\%) & 0 \\
\hline \multicolumn{3}{|l|}{ Any drug-related adverse event ${ }^{\mathrm{a}}$} \\
\hline Possible relationship & $29(22 \%)$ & $5 \mathrm{I}(39.5 \%)$ \\
\hline Probable/likely relationship & $24(18.2 \%)$ & $29(22.5 \%)$ \\
\hline Certain relationship & $\mathrm{I}(0.8 \%)$ & $5(3.9 \%)$ \\
\hline $\begin{array}{l}\text { Any adverse event causing premature } \\
\text { discontinuation from trial }\end{array}$ & $6(4.5 \%)$ & $8(6.2 \%)$ \\
\hline $\begin{array}{l}\text { Time to premature withdrawal due } \\
\text { to adverse event }(h)^{\mathrm{b}}\end{array}$ & $12.1 \pm 9.5$ & $11.5 \pm 9.5$ \\
\hline \multicolumn{3}{|l|}{$\begin{array}{l}\text { Any adverse event } \geq 5 \% \text { incidence } \\
\text { in either group }\end{array}$} \\
\hline Nausea & $34(25.8 \%)$ & 47 (36.4\%) \\
\hline Dizziness & 21 (I5.9\%) & $24(18.6 \%)$ \\
\hline Somnolence & $12(9.1 \%)$ & $18(14.0 \%)$ \\
\hline Vomiting & $9(6.8 \%)$ & $16(12.4 \%)$ \\
\hline Sweating increased & $6(4.5 \%)$ & $9(7.0 \%)$ \\
\hline
\end{tabular}

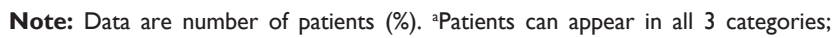
${ }^{\mathrm{b}}$ Calculated for full analysis set; 'Listed in order of decreasing frequency in the tramadol/paracetamol group.
One 19-year-old female receiving tramadol/paracetamol experienced 2 serious AEs of severe intensity (nausea and hypotension) 3.5 hours after intake of trial medication leading to hospitalization for 1 night. Both events resolved within the next day. The patient continued taking the trial medication.

Six $(4.5 \%)$ patients receiving tramadol/paracetamol and $8(6.2 \%)$ patients on tramadol prematurely withdrew owing to AEs; the most common reasons were gastrointestinal complaints. Time to premature discontinuation due to AEs was calculated for the FAS population and similar for both treatments: $12.1 \pm 9.5$ hours for tramadol/paracetamol and $11.5 \pm 9.5$ hours for tramadol (Table 3 ).

There were no differences between treatments regarding vital signs and sedation assessment. Ten minutes after deflation of the tourniquet, the majority of patients in both groups were awake and spontaneous communication was possible (93.8\% for tramadol/paracetamol, $91.4 \%$ for tramadol; FAS).

\section{Discussion}

This randomized, double-blind, multicenter trial demonstrated that postoperative pain following ambulatory hand surgery was effectively managed in the majority of patients by tramadol $37.5 \mathrm{mg} /$ paracetamol $325 \mathrm{mg}$ combination treatment with no statistically significant differences in efficacy to tramadol $50 \mathrm{mg}$ monotherapy but with fewer AEs.

Intake of tramadol/paracetamol combination tablets before and immediately after surgery and every 6 hours thereafter markedly reduced pain intensity from surgery day by the end of the first postoperative day. A pain intensity score $\leq 3$ (on a numerical rating scale up to 10 for 'worst imaginable pain') was reported by $84 \%$ of these patients and the number of patients experiencing no pain $($ score $=0$ ) more than doubled from $16 \%$ on the day of surgery to $36 \%$ on the first postoperative day. Accordingly, treatment satisfaction was high; 78\% were considered treatment responders based on their satisfaction with the medication. Good efficacy was also confirmed by the low proportion of patients resorting to rescue medication and the low premature withdrawal rate owing to a lack of efficacy.

Most of the published literature on efficacy of the combination tablet in the management of postoperative pain report the outcome of single-dose treatment for moderate to severe dental pain. ${ }^{12,27-29}$ In a meta-analysis of 5 randomized, doubleblind, placebo-controlled trials the number-needed-to-treat (NNT) to achieve at least 50\% pain relief over 6 hours was 2.6 for tramadol $75 \mathrm{mg} /$ paracetamol $650 \mathrm{mg}$, which was superior to an NNT of 9.9 for tramadol $75 \mathrm{mg}$, and 3.6 for paracetamol $650 \mathrm{mg} .{ }^{12}$ Relative benefits of the three single-dose analgesic treatments were similar when pooled 
data from gynecological and orthopedic procedures were analyzed. ${ }^{12}$ Efficacy of multiple tramadol $37.5 \mathrm{mg} /$ paracetamol $325 \mathrm{mg}$ doses proved superior to placebo following abdominal or orthopedic inpatient surgery and pain relief was similar to the combination of codeine $60 \mathrm{mg} /$ paracetamol $600 \mathrm{mg}$ during the immediate postoperative period. ${ }^{13}$

In the present trial, analgesic efficacy of the two treatments was comparable but multiple-dose tramadol/paracetamol treatment showed a better safety profile than tramadol monotherapy. This is in line with a short-term (10 days) treatment trial in patients with subacute low back pain which reported significantly fewer AEs under combination therapy. ${ }^{17}$ In the present trial, the overall incidence of AEs was markedly lower in the combination group with significantly fewer patients reporting AEs. The majority of AEs in both groups were side-effects usually associated with tramadol such as nausea, dizziness, somnolence, vomiting, and increased sweating. ${ }^{10}$ Gastrointestinal and nervous system disorders were reported with a $15.4 \%$ and $11.4 \%$ lower frequency, respectively, for tramadol/paracetamol treatment.

The difference in tolerability might thus be attributed to the reduced intake of tramadol in the combination group which was $23.8 \%$ less than in patients receiving tramadol monotherapy. Tramadol-sparing did not lower the analgesic efficacy of the combination tablet, probably owing to the individual compounds acting additively. ${ }^{8,9}$

In summary, tramadol/paracetamol combination tablets provided comparable analgesic efficacy to tramadol capsules, with fewer AEs, in patients experiencing postoperative pain following ambulatory hand surgery.

\section{List of study investigators}

Belgium: V Macquaire, E Spyropoulos; France: A Babinet, RP Bleton, P Catoire, M Chauvin, L Delaunay, E Gaertner, L Le Gourrier, R Lepescu, C Menigaux, B Toussaint; Germany: W Bannasch, R Finck, J Freier, D Petermeise, E Pinkowski, R Pinkowski; Italy: M Berti, M Montebugnoli; Portugal: R Costa, C da Silva João, N Dias, P Lemos, D Marques, MF Martins Nunes, JC Parreira; Spain: E Catalá, E Concha, J de Andrés, FJ González-Carrasco, A Garcia-Muret, D Román, LM Torres; Sweden: H Alnehill, A Amilon, N Rawal; Switzerland: G Schüpfer, M Wietlisbach, S Zbinden; The Netherlands: HPM Renkens, E van Ark, RL van Leersum.

\section{Acknowledgments}

The authors would like to thank all participating investigators. The trial was sponsored by Grünenthal GmbH, Aachen, Germany. Editorial assistance and publication coordination were provided by E. Grosselindemann and B. Brett (Brett Medical Writing). All associated costs were met by the sponsor of the trial.

\section{Disclosure}

NR has received honoraria and speaker fees from Baxter, Nycomed, Vygon, Sintetica, and MSD. EC has received speaker fees from Grünenthal. All other authors have nothing to declare.

\section{References}

1. Aylin P, Williams S, Jarman B, Bottle A. Trends in day surgery rates. BMJ. 2005;331:803.

2. Cullen KA, Hall MJ, Golosinskiy A. Ambulatory surgery in the United States, 2006. National Health Statistics Reports 2009. http://www.cdc. gov/nchs/products/nhsr.htm. Accessed November 10, 2010.

3. Rawal N. Analgesia for day-case surgery. Br J Anaesth. 2001;87:73-87.

4. Chung F, Ritchie E, Su J. Postoperative pain in ambulatory surgery. Anesth Analg. 1997;85:808-816.

5. Gold BS, Kitz DS, Lecky JH, Neuhaus JM. Unanticipated admission to the hospital following ambulatory surgery. JAMA. 1989;262:3008-3010.

6. Rawal N, Hylander J, Nydahl P-A, Olofsson I, Gupta A. Survey of postoperative analgesia following ambulatory surgery. Acta Anaesthesiol Scand. 1997;41:1017-1022.

7. Beauregard L, Pomp A, Choinière M. Severity and impact of pain after day-surgery. Can J Anaesth. 1998;45:304-311.

8. Raffa RB. Pharmacology of oral combination analgesics: rational therapy for pain. J Clin Pharm Ther. 2001;26:257-264.

9. Desmeules J, Rollason V, Piguet V, Dayer P. Clinical pharmacology and rationale of analgesic combinations. Eur JAnaesthesiol. 2003; 20(Suppl 28):7-12.

10. Scott LJ, Perry CM. Tramadol. A review of its use in perioperative pain. Drugs. 2000;60:39-176.

11. Parfitt K, editor. Analgesics, anti-inflammatory drugs and antipyretics. In: Martindale, The Complete Drug Reference, 32nd edition. London, UK: Pharmaceutical Press; 1999:1-91.

12. Edwards JE, McQuay HJ, Moore RA. Combination analgesic efficacy: individual patient data meta-analysis of single-dose oral tramadol plus acetaminophen in acute postoperative pain. J Pain Symptom Manage. 2002;23:121-130.

13. Smith AB, Ravikumar TS, Kamin M, Jordan D, Xiang J, Rosenthal N, on behalf of the CAPSS-115 Study Group. Combination tramadol plus acetaminophen for postsurgical pain. Am J Surg. 2004;187:521-527.

14. Bennett RM, Kamin M, Karim R, Rosenthal N. Tramadol and acetaminophen combination tablets in the treatment of fibromyalgia pain: a double-blind, randomized, placebo-controlled study. Am J Med. 2003;114:537-545.

15. Ruoff GE, Rosenthal N, Jordan D, Karim R, Kamin M, on behalf of the Protocol CAPSS-112 Study Group. Tramadol/acetaminophen combination tablets for the treatment of chronic lower back pain: a multicenter, randomized, double-blind, placebo-controlled outpatient study. Clin Ther. 2003;25:1123-1141.

16. Peloso PM, Fortin L, Beaulieu A, Kamin M, Rosenthal NR on behalf of the Protocol TRP-CAN-1 Study Group. Analgesic efficacy and safety of tramadol/acetaminophen combination tablets $\left(\right.$ Ultracet $\left.^{\circledR}\right)$ in treatment of chronic low back pain: a multicenter, outpatient, randomized, double blind, placebo controlled trial. J Rheumatol. 2004;31:2454-2463.

17. Perrot S, Krause D, Crozes P, Naïm C, for the GRTF-ZAL-1 Study Group. Efficacy and tolerability of paracetamol/tramadol $(325 \mathrm{mg} / 37.5 \mathrm{mg})$ combination treatment compared with tramadol $(50 \mathrm{mg})$ monotherapy in patients with subacute low back pain: a multicenter, randomized, double-blind, parallel-group, 10-day treatment study. Clin Ther. 2006;28:1592-1606. 
18. Silberstein SD, Freitag FG, Rozen TD, Kudrow DB, Hewitt DJ, Jordan DM, et al. Tramadol/acetaminophen for the treatment of acute migraine pain: findings of a randomized, placebo-controlled trial. Headache. 2005;45:1317-1327.

19. Silverfield JC, Kamin M, Wu SC, Rosenthal N, for the CAPSS-105 Study Group. Tramadol/acetaminophen combination tablets for the treatment of osteoarthritis flare pain: a multicenter, outpatient, randomized, double-blind, placebo-controlled, parallel-group, add-on study. Clin Ther. 2002;24:282-297.

20. Lee EY, Lee EB, Park BJ, Lee CK, Yoo B, Lim MK, et al. Tramadol 37.5-mg/acetaminophen 325-mg combination tablets added to regular therapy for rheumatoid arthritis pain: a 1-week, randomized, doubleblind, placebo-controlled trial. Clin Ther. 2006;28:2052-2060.

21. Dhillon S. Tramadol/paracetamol fixed-dose combination. A review of its use in the management of moderate to severe pain. Clin Drug Investig. 2010;30:711-738.

22. Ko SH, Kwon HS, Yu JM, Baik SH, Park IB, Lee JH, et al. Comparison of the efficacy and safety of tramadol/acetaminophen combination therapy and gabapentin in the treatment of painful diabetic neuropathy. Diabet Med. 2010;27:1033-1040.

23. Brull TR, von Schroeder H, Anastakis D, Graham B, Katz J, Chan VWS, et al. Regional anesthesia versus general anesthesia for ambulatory hand surgery. Reg Anesth Pain Med. 2003;28:A1(abstract).
24. Pregler JL, Kapur PA. The development of ambulatory anesthesia and future challenges. Anesthesiol Clin North America. 2003;21: 207-228.

25. Rawal N, Allvin R, Amilon A, Ohlsson T, Hallén J. Postoperative analgesia at home after ambulatory hand surgery: a controlled comparison of tramadol, metamizol, and paracetamol. Anesth Analg. 2001;92: 347-351.

26. Camporesi EM, Greeley WJ, Lumb PD, Watkins WD. Anesthesia. In: Sabiston DC Jr, editor. Textbook of Surgery. WB Saunders Company; 1991:148-163.

27. Fricke JR, Karim R, Jordan D, Rosenthal N. A double-blind, singledose comparison of the analgesic efficacy of tramadol/acetaminophen combination tablets, hydrocodone/acetaminophen combination tablets, and placebo after oral surgery. Clin Ther. 2002;24:953-968.

28. Fricke JR, Hewitt DJ, Jordan DM, Fisher A, Rosenthal NR. A doubleblind placebo-controlled comparison of tramadol/acetaminophen and tramadol in patients with postoperative dental pain. Pain. 2004;109: 250-257.

29. Jung Y-S, Kim DK, Kim M-K, Kim H-J, Cha I-H, Lee E-W. Onset of analgesia and analgesic efficacy of tramadol/acetaminophen and codeine/acetaminophen/ibuprofen in acute postoperative pain: a singlecenter, single-dose, randomized, active-controlled, parallel-group study in a dental surgery pain model. Clin Ther. 2004;26:1037-1045.
Journal of Pain Research

\section{Publish your work in this journal}

The Journal of Pain Research is an international, peer-reviewed, open access, online journal that welcomes laboratory and clinical findings in the fields of pain research and the prevention and management of pain. Original research, reviews, symposium reports, hypothesis formation and commentaries are all considered for publication.

\section{Dovepress}

The manuscript management system is completely online and includes a very quick and fair peer-review system, which is all easy to use. Visit http://www.dovepress.com/testimonials.php to read real quotes from published authors. 